\title{
Targeting Immunometabolism Mediated by CD73 Pathway in EGFR-Mutated Non-small Cell Lung Cancer: A New Hope for Overcoming Immune Resistance
}

\author{
Anna Passarelli ${ }^{1 * \dagger}$, Michele Aieta ${ }^{1 \dagger}$, Alessandro Sgambato ${ }^{2}$ and Cesare Gridelli ${ }^{3}$ \\ ${ }^{1}$ Unit of Medical Oncology, Department of Onco-Hematology, Centro di Riferimento Oncologico della Basilicata \\ (IRCCS-CROB), Rionero in Vulture, Italy, ${ }^{2}$ Laboratory of Pre-clinical and Translational Research, Centro di Riferimento \\ Oncologico della Basilicata (IRCCS-CROB), Rionero in Vulture, Italy, ${ }^{3}$ Division of Medical Oncology, "S. G. Moscati" Hospital, \\ Avellino, Italy
}

OPEN ACCESS

Edited by:

Peng Qu,

National Institutes of Health $(\mathrm{NIH})$,

United States

Reviewed by:

Hua Jiang,

Indiana University-Purdue University Indianapolis, United States

Jing HaO,

Shandong University, China

*Correspondence:

Anna Passarelli

passarellian@libero.it;

anna.passarelli@crob.it

†These authors have contributed equally to this work

Specialty section:

This article was submitted to Cancer Immunity and Immunotherapy, a section of the journal

Frontiers in Immunology

Received: 10 May 2020

Accepted: 08 June 2020

Published: 14 July 2020

Citation:

Passarelli A, Aieta M, Sgambato A

and Gridelli C (2020) Targeting Immunometabolism Mediated by CD73 Pathway in EGFR-Mutated

Non-small Cell Lung Cancer: A New

Hope for Overcoming Immune

Resistance. Front. Immunol. 11:1479.

doi: 10.3389/fimmu.2020.01479
Despite the relevant antitumor efficacy of immunotherapy in advanced non-small cell lung cancer (NSCLC), the results in patients whose cancer harbors activating epidermal growth factor receptor (EGFR) mutations are disappointing. The biological mechanisms underlying immune escape and both unresponsiveness and resistance to immunotherapy in EGFR-mutant NSCLC patients have been partially investigated. To this regard, lung cancer immune escape largely involves high amounts of adenosine within the tumor milieu with broad immunosuppressive effects. Indeed, besides immune checkpoint receptors and their ligands, other mechanisms inducing immunosuppression and including adenosine produced by ecto-nucleotidases CD39 and CD73 contribute to lung tumorigenesis and progression. Here, we review the clinical results of immune checkpoint inhibitors in EGFR-mutant NSCLC, focusing on the dynamic immune composition of EGFR-mutant tumor microenvironment. The adenosine pathway-mediated dysregulation of energy metabolism in tumor microenvironment is suggested as a potential mechanism involved in the immune escape process. Finally, we report the strong rationale for planning strategies of combination therapy with immune checkpoints blockade and adenosine signaling inhibition to overcome immune escape and immunotherapy resistance in EGFR-mutated NSCLC.

Keywords: non-small cell lung cancer, epidermal growth factor receptor, immune metabolism, adenosine, CD73, immunotherapy, immune resistance

\section{INTRODUCTION}

Primary lung cancer is the most common malignant tumor and the main cause of cancer-related death in the world (1). Non-small cell lung cancer (NSCLC) accounts for $80-90 \%$ of lung cancers, while small cell lung cancer (SCLC) has decreased in terms of frequency over the past decades $(2,3)$. The World Health Organization (WHO) calculates that lung cancers cause 1.5 million deaths per years and about $70 \%$ of them are due to smoking. However, recent advances in the field of anti-cancer therapies and mutations in oncogenic drivers (4), such as Epidermal Growth Factor Receptor (EGFR) mutations and Anaplastic Lymphoma Kinase (ALK) gene translocation, have 
improved the outlook in terms of both progression-free survival (PFS) and overall survival (OS), showing a promising future for advanced NSCLC therapy (5).

Tyrosine kinase inhibitors (TKIs) in oncogene-driven tumors and immunotherapy are the two major evolving strategies in the treatment of $\operatorname{NSCLC}(6,7)$. To date, EGFR-TKIs are recommended by clinical guidelines as optimal first-line strategy in EGFR-mutated NSCLC (8). Despite initial responsiveness to EGFR-TKIs, acquired resistance within 9-18 months is almost inevitable (9). Therefore, the onset of acquired resistance to EGFR-TKIs has raised hopes of a role for immune checkpoint inhibitors (ICIs) characterized instead by durable response (10).

The use of immune checkpoint inhibitors (ICIs) has revolutionized the management of patients with non-oncogene addicted NSCLC in both first- and second-line settings, showing an unexpected long-term effectiveness and a good toxicity profile (11); on the other hand, to date the clinical outcomes of ICIs in oncogene-addicted NSCLC are disappointing.

To this regard, recent clinical studies have described limited efficacy of ICIs, targeting mainly cytotoxic T-lymphocyte antigen-4 (CTLA-4), programmed-cell death-1 (PD-1) or its ligand PD-L1, in NSCLC harboring EGFR mutations and TKIs naive (12). In addition, a recent work has suggested that the different molecular features of EGFR mutations in NSCLC may lead to a different responsiveness and outcomes to ICIs (13). It is noteworthy that EGFR-TKIs could modulate immune responsiveness to cancer by shaping the tumor microenvironment (TME) and enhancing ICIs benefit (14). Therefore, several clinical trials evaluating both efficacy and safety of immunotherapy combined with targeted therapy in patients EGFR-mutant NSCLC are currently ongoing $(15,16)$.

To date, several evidence have suggested that EGFR-mutated cancer cells represent a crucial hallmark of immunosuppression (17), actively establishing an immunosuppressive milieu and negatively influencing the quality of T-cell immune response.

Interestingly the immunosuppressive effects of EGFR mutations could also result in an immune metabolic dysfunction, in which an emerging role seems to be played by CD39/CD73 ectonucleotidases, catalyzing both over-production and release of extracellular adenosine (ADO), known as a powerful immunosuppressive nucleoside (18). In specific, the immune metabolic reprograming mediated by adenosine signaling in TME is reported as a further hallmark of EGFR-mutated NSCLC, with the precise aim to evade the immune surveillance and induce innate immune resistance to ICIs (19).

In this review, we discuss the role and features of TME in NSCLC harboring EGFR-mutation focusing on the involvement of immunometabolism mechanisms mediated by CD39/CD73adenosine signaling. The potential application of targeting this pathway in the therapeutic strategy for overcoming the immunotherapy resistance is also evaluated.

\section{IMMUNOTHERAPY IN THE MANAGEMENT OF EGFR-MUTATED LUNG CANCER}

The relevant success of the immunotherapy associated with favorable safety profile in advanced lung cancer treatment
(20-23) suggests that, similarly to other cancer types, escape, or immune evasion processes concur to lung cancer pathogenesis and progression as well $(24,25)$. Indeed, monoclonal antibodies (mAb)s targeting PD-1, PD-L1, and CTLA-4 immune checkpoints leading to increased anti-tumor response due to increased T-cell activity and proliferation, have received regulatory approval across a wide range of tumors, including NSCLC (26). Specifically, patients with PD-L1 tumor proportion score (TPS) $\geq 50 \%$ are typically offered monotherapy with the anti-PD-1 mAb (Pembrolizumab ${ }^{\circledR}$ ) (27). For patients with PD-L1 expression $<50 \%$, the combination of a platinum-doublet chemotherapy and Pembrolizumab has been recently approved (28). In addition, the combination of carboplatin plus paclitaxel with anti-angiogenic drug (Bevacizumab ${ }^{\circledR}$ ) and anti-PD-L1 $\mathrm{mAb}$ (Atezolizumab ${ }^{\circledR}$ ) represents an alternative treatment for patients with non-squamous NSCLC, which just received EMA and FDA approval (FDA approval excludes patients with EGFR or $A L K$ genomic tumor aberrations) (29). In the second-line setting, rather than single-agent chemotherapy, Pembrolizumab has been approved for tumors that express PD-L1 (30), while Nivolumab and Atezolizumab represent a standard option regardless of tumor PD-L1 expression (31-33).

Despite promising advances in immunotherapy, the role of ICIs in oncogene-addicted NSCLC remains unclear and conflicting. The majority of data come from subgroup analyses with low number of patients, therefore the use of ICIs, when permitted by regulatory agencies, should only be considered when other available therapies, including standard EGFR-TKIs, fail (34).

Unfortunately, after failure of first-line TKIs, patients with EGFR mutations have limited treatment options. Two metaanalysis covering several clinical trials observed relatively poor efficacy and low response rates to PD-1/PD-L1 inhibitors vs. standard second-line chemotherapy among patients with pretreated EGFR-mutant lung cancer $(35,36)$. Based on molecular status, the OS improvement was confirmed for EGFR wild-type lung cancers (OS hazard ratio (HR): $0.67 ; p<0.001$ ], but not in those EGFR mutated (OS HR: $1.11 ; p=0.54$ ), although no clear conclusions can be drawn due to the limited number of patients as part of subgroup analyses.

Of most interest, Lisberg et al. reported the role of immunotherapy with Pembrolizumab as first-line for EGFRmutant NSCLC patients with PD-L1 expression of at least $1 \%$, confirming once again the failure of immunotherapy alone for EGFR mutant patients (12). Although this trial evaluated Pembrolizumab in only 10 EGFR-mutant, TKI naïve patients, the lack of efficacy in terms of objective response reported was striking, especially since $70 \%$ of these patients had PD-L1 expression $\geq 50 \%(12,37)$. About this, an interesting retrospective analysis showed that among EGFR-mutated NSCLC patients with high PD-L1 expression (TPS $\geq 50 \%$ ) the efficacy of PD-1 inhibitors tended to be lower as compared to EGFR wild-type patients (38). These disappointing results could be related, at least partly, to the genomic landscape of EGFR-mutant NSCLC (39). Indeed, this type of cancer shapes a typical "uninflamed" TME characterized by a lack of T-cell infiltration, a shrinking proportion of $\mathrm{PD}-\mathrm{L}^{+} / \mathrm{CD}^{+}$tumor infiltrating lymphocytes (TILs), an immune metabolic reprogramming process and a 
lower mutation burden (17). Therefore, the limited benefit of immunotherapy in EGFR-mutant patients has led to alternative approaches or rather combination strategies targeting several pathways. These include ICIs plus an anti-angiogenic therapy or EGFR-TKIs (Table 1), as well as ICIs with chemotherapy to increase immunogenicity of EGFR-mutant tumors and responsiveness to ICIs (40).

Importantly, the IMpower150 trial reported that in EGFRmutant NSCLC patients the addition of Atezolizumab to Bevacizumab plus carboplatin plus paclitaxel (ABCP) provided significant clinical benefit (41). In specific, in a subset analysis of EGFR-mutant patients treated after TKI failure, median OS was not estimable for patients treated with the addition of ABCP vs. 17.5 months for patients treated with Bevacizumab, carboplatin plus paclitaxel (BCP) (HR 0.31; 95\% CI 0.11-0.83); median PFS was 10.3 months with ABCP vs. 6.1 months with BCP, associated with a similar and good safety profile (39). However, the subgroup analysis and the very low patients number (overall 114 patients) with activating EGFR mutation or EML4-ALK rearrangement status are important limitations.

Furthermore, preclinical studies reported an immune modulatory effect of EGFR signaling by regulating expression of MHC I/II and PD-L1 on tumor cells and the activity of T-cells. This suggests a potential synergistic effect for the use of immunotherapy in combination with EGFR-TKIs (42), according to the recent evidence of long-lasting antitumor responses of $B R A F / M E K$ inhibitors with immunotherapy in the treatment of $B R A F$-mutated metastatic melanoma (43). However, this promising combination strategy remains controversial due to the significant toxicity observed in several clinical trials following administration of anti-PD-(L) $1 \mathrm{mAbs}$ in combination with EGFR-TKIs $(44,45)$ (see Table 1).

In conclusion, all these studies suggest that further and prospective clinical trials with different and less toxic drugs are required to better define if there is a role for ICIs strategy in the treatment of oncogene-addicted NSCLC.

\section{THE IMMUNE MICROENVIRONMENT IN EGFR-MUTANT NSCLC}

The TME composition plays a considerable role in tumor growth and progression (24). Surprisingly, the TME is able to act as either obstacle or facilitator of cancer proliferation and progression by affecting several biological mechanisms. During tumorigenesis, both immune and EGFR-mutant tumor cells are subjected to the immunoediting process consisting of dynamic and interconnected phases, including elimination, equilibrium, and finally immune evasion. Therefore, this complex interplay is essential to define appropriate strategies to target TME as part of the anti-cancer therapy.

To this regard, recently it has been reported that the TME of EGFR-mutated NSCLC concurs to create an immunosuppressive milieu, as represented in Figure 1 (46). In fact, immunosuppressive effects of EGFR mutations shape both composition and function of TME by interfering with several intracellular pathways and modulating immune accessory cells such as tumor-infiltrating lymphocytes (TILs), natural killer (NK) cells, T-regulatory cells (Tregs), myeloid-derived suppressor cells (MDSCs), tumor-associated macrophages (TAMs), involved in the increased release of immunoregulatory soluble factors such as cytokines and exosomes, as summarized in Table $2(47,48)$.

In NSCLC, several studies reported that highly infiltrating Tlymphocytes in TME are related to the efficacy of immunotherapy and thus good prognosis (49). Indeed, several studies found significantly reduced $\mathrm{CD}^{+}$TILs in an EGFR-mutated NSCLC group compared with a wild-type EGFR group (17). In terms of

TABLE 1 | Clinical trials of immunotherapy combined with EGFR-TKi in EGFR-mutated NSCLC.

\begin{tabular}{|c|c|c|c|c|c|c|c|}
\hline \multirow[b]{2}{*}{ Clinical trial } & \multicolumn{2}{|c|}{ Drugs Combination } & \multirow[b]{2}{*}{ Condition or disease } & \multirow[b]{2}{*}{ Phase } & \multirow{2}{*}{$\begin{array}{l}\text { Outcome } \\
\text { ORR (\%) }\end{array}$} & \multirow{2}{*}{$\begin{array}{c}\text { Safety } \\
\text { AEs Grade 3-4 }\end{array}$} & \multirow[b]{2}{*}{ Trial Status } \\
\hline & TKi & ICI & & & & & \\
\hline NCT02088112 & Gefitinib & Durvalumab & $\begin{array}{l}\text { EGFRm NSCLC, TKi } \\
\text { naive }\end{array}$ & 1 & $63 \%$ & $58 \%$ & $\begin{array}{l}\text { Active, } \\
\text { not recruiting }\end{array}$ \\
\hline NCT02013219 & Erlotinib & Atezolizumab & $\begin{array}{l}\text { EGFRm NSCLC, TKi } \\
\text { naïve or previously } \\
\text { treated (without TKi) }\end{array}$ & I & $75 \%$ & $50 \%$ & $\begin{array}{l}\text { Active, } \\
\text { not recruiting }\end{array}$ \\
\hline- & Erlotinib & Nivolumab & $\begin{array}{l}\text { EGFRm NSCLC, TKi } \\
\text { naïve or treated }\end{array}$ & I/II & $15 \%$ & $25 \%$ & Completed \\
\hline $\begin{array}{l}\text { NCT02143466 } \\
\text { TATTON }\end{array}$ & Osimertinib & Durvalumab & $\begin{array}{l}\text { EGFRm NSCLC, TKi } \\
\text { naïve or treated }\end{array}$ & I & $43 \%$ & 48\% (ILD-transaminitis) & $\begin{array}{l}\text { Active, } \\
\text { not recruiting }\end{array}$ \\
\hline $\begin{array}{l}\text { NCT02454933 } \\
\text { CAURAL }\end{array}$ & Osimertinib & Durvalumab & $\begin{array}{l}\text { EGFR-T790M NSCLC } \\
\text { previously treated with } \\
\text { TKi }\end{array}$ & III & $64 \%$ & $8 \%$ & $\begin{array}{l}\text { Active, } \\
\text { not recruiting }\end{array}$ \\
\hline NCT02039674 & Erlotinib/Gefitinib & Pembrolizumab & EGFRm NSCLC & I/II & - & - & $\begin{array}{l}\text { Active, } \\
\text { not recruiting }\end{array}$ \\
\hline
\end{tabular}

Source from ClinicalTrials.gov.

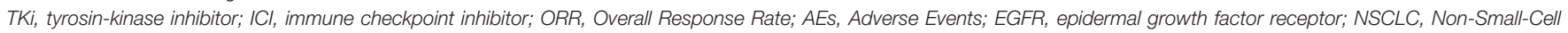
Lung Cancer; N/A, not available; ILD, Interstitial Lung Disease. 




FIGURE 1 | The tumor microenvironment in EGFR-addicted NSCLC. EGFR-mutated NSCLC is typically characterized by an "uninflamed" tumor microenvironment, immunological tolerance, and weak immunogenicity. Recently, it was suggested that the over-expression of CD39/CD73-adenosine signaling also induces an immunosuppressive TME. Indeed, CD39/CD73 ectonucleotidases are widely expressed by lung cancer cells and induce the high extracellular production and release of immunosuppressive adenosine that shapes the activity of innate and adaptive immune system cells and endothelial cells in TME. Specifically, the activation of CD39 induces the de-phosphorylation of ATP to ADP and, subsequently, to AMP, while CD73 catalyzes the hydrolysis of AMP into adenosine and phosphate. By binding the A2A adenosine receptor A2AR, the most common receptor subtype expressed by both adaptive and innate immunity, extracellular adenosine induces inhibitory signals in TME that restrain the activity of immune system cells, promoting growth and survival of EGFR-mutated lung cancer cells. In addition, exosomes derived by tumor cells also contribute to modulate immunosuppression by influencing PD-L1+/CD73+ expression and extracellular adenosine release. EGFR, Epidermal growth factor receptor; Tregs, T regulatory T-cells; MHC, Major histocompatibility complex; PD-1, Programmed cell death protein; CTLA-4, Cytotoxic T lymphocyte antigen-4; MDSCs, Myeloid-derived suppressor cells; TCR, T-cell receptor; VEGF, Vascular endothelial growth factor; IL, Interleukin; M2, Macrophages 2; ATP, Adenosine triphosphate; ADP, Adenosine diphosphate; AMP, Adenosine monophosphate; CCL2, C-C motif chemokine ligand 2; FoxP3, forkhead box P3.

EGFR mutation sites different immunological profiles have been reported with the prevalence of "inflamed" TME consisting of higher level of functional TILs in $E G F R^{L 858 R}$ samples compared to EGFR exon 19 deletion tumor samples (50).

To support the evidence on the heterogeneity of EGFR mutations, Hastings et al. retrospectively analyzed clinical and molecular data on 171 cases of EGFR-mutant lung tumors treated with ICIs. Although EGFR-mutant tumors typically showed a low response to immunotherapy, clinical outcomes appear to vary by allele. In specific, $E G F R^{L 858 R}$ tumors had a similar response rate and OS to an EGFR wild-type NSCLC population, while tumors harboring deletions in exon 19 cases did substantially worse (13).

Furthermore, it is noteworthy that the relationship between EGFR mutations and PD-L1 expression remains largely controversial, since pre-clinical data reported that the activation of EGFR signaling directly drives "intrinsic" PD-L1 upregulation in a NSCLC model through several pathways such as PI3K/AKT/mTOR, Ras/RAF/MEK/ERK, JAK/STAT, and NF-kB (39). In addition, the activation of EGFR signaling may lead to the down-regulation of both class I and II antigens of the major histocompatibility complex (MHC) whose expression is regulated by the MEK/ERK pathway (51). Conversely, a large number of clinical trials reported that PD-L1 expression in EGFR wild-type tumors was significantly higher than in EGFR mutant NSCLC (52).

Regarding the field of predictive biomarkers research in NSCLC and other cancer types, tumor mutation burden (TMB) also emerged as a promising predictive biomarker of ICIs efficacy (53). TMB is the total number of insertion, deletion, and substitution mutations per megabase of the coding region of a tumor genome. Among patients with NSCLC treated with anti-PD-1 mAb, higher non-synonymous TMB seemed to be related with greater benefit, but a large phase III randomized trial did not confirm these preliminary observations (54). However, EGFR-addicted NSCLC were shown to have also low TMB (55).

Further, the activation of the EGFR oncogene pathway induces the release of several immunosuppressive factors to accomplish evasion of the host anti-cancer immune response. On the other hand, the inefficient killing of tumor cells is mostly due to a direct effect of EGFR-mutated lung cells through the over-production of negative modulators of immune cells including tumor necrosis factor- $\beta$ (TGF- $\beta$ ), interleukin-10 (IL-10), vascular endothelial 
TABLE 2 | The major hallmarks and mechanisms of Tumor Immune Escape in the TME of EGFR-mutated non-small cell lung cancer.

\begin{tabular}{|c|c|c|}
\hline Mechanism of action & Population(s)/pathway(s) & Effect(s) on TME \\
\hline Tumor-infiltrating lymphocytes (TIL)s & $\begin{array}{l}\mathrm{CD}^{+} \text {Iymphocytes } \\
\text { CD8 }{ }^{+} \text {T-cells } \\
\text { CD4 }{ }^{+} \text {T-cells }\end{array}$ & Inefficacy tumor-cytotoxicity of T-cells \\
\hline $\begin{array}{l}\text { Over-expression of inhibitory immune } \\
\text { checkpoints }\end{array}$ & $\begin{array}{l}\uparrow \text { CTLA-4, PD-1, LAG3, TIM3, VISTA, } \\
\text { TIGIT }\end{array}$ & Inhibition of T-cell function/T-cell anergy \\
\hline $\begin{array}{l}\text { Over-expression of immune checkpoint } \\
\text { ligands }\end{array}$ & $\begin{array}{l}\text { PD-L1 } \\
\text { PD-L2 }\end{array}$ & Activation of inhibitory immune checkpoints \\
\hline Up-regulation of immune suppressive cells & $\begin{array}{l}\text { MDSCs } \\
\text { CD4 } 4^{+} \text {CD25 } 5^{+} \mathrm{FoxP}^{+} \text {Tregs } \\
\text { Macrophage infiltration (M2-TAM) }\end{array}$ & $\begin{array}{l}\text { Inhibition of T-cell function } \\
\text { Direct pro-tumorigenic effect (VEGF, TGF- } \beta \text { ) }\end{array}$ \\
\hline Intracellular activation of EGFR signaling & $\begin{array}{l}\uparrow \text { JAK/STAT3 } \\
\uparrow \text { PIIK/AKT/mTOR } \\
\uparrow \text { Ras/RAF/MEK/ERK } \\
\uparrow \text { NF-KB }\end{array}$ & $\begin{array}{l}\downarrow \text { Down-regulation of MHC I/II class }-\downarrow \text { Inhibition of STAT1 activity } \\
\uparrow \text { MDSCs }-\downarrow \text { DCs and APCs - } \uparrow \text { TAM-M2 polarization } \\
\uparrow / \downarrow \text { PD-L1; } \downarrow \text { IFN } \gamma \text { signature } \\
\uparrow \text { Production and release of negative modulators (TGF- } \beta \text {, IDO, CCL-2) }\end{array}$ \\
\hline Tumor mutational load and Neoantigens & $\downarrow \mathrm{TMB}$ & $\begin{array}{l}\text { Low TMB may negatively influence the immune-mediated anti-tumor } \\
\text { response }\end{array}$ \\
\hline Dysregulation of the immunometabolism & $\begin{array}{l}\uparrow \mathrm{CD} 39 / \mathrm{CD} 73 \text { - adenosine signaling } \uparrow \\
\text { IDO } \uparrow \mathrm{ARG}-1\end{array}$ & $\begin{array}{l}\uparrow \text { Immunosuppressive TME restraining anti-tumor immunity through } \\
\text { A2AR } \\
\uparrow \text { Degradation of tryptophan into immunosuppressive kynurenines } \\
\uparrow \text { TAM-M2 polarization }\end{array}$ \\
\hline
\end{tabular}

TME, tumor microenvironment; mAb, monoclonal antibody; EGFR, epidermal growth factor receptor; NSCLC, Non-Small-Cell Lung Cancer; MHC, major histocompatibility complex; CTLA4, cytotoxic T-lymphocyte antigen 4; PD-1, programmed death 1; LAG3, lymphocyte activation gene-3; TIM3, T cell immunoglobulin and mucin domain 3; VISTA, V-domain immunoglobulin suppressor of T-cell activation; PDL1, programmed death ligand 1; PDL2, programmed death ligand 2; IFN, interferon; MDSCS, myeloid-derived suppressor cells; Tregs, regulatory T cells; TAM, tumor-associated macrophages; MMP, matrix metalloproteinase; CCL-2, CC chemokine ligand-2; TGF, tumor necrosis factor; VEGF, vascular endothelial growth factor; iNOS, inducible nitric oxide synthase; IDO, indoleamine 2,3 dioxygenase; IL, interleukin; TMB, tumor mutation burden; A2AR, adenosine 2A receptor; ARG, arginase.

growth factor (VEGF), indolamine 2,3-dioxygenase (IDO), C-C chemokine ligand 2 (CCL-2), arginase (ARG)-1, and adenosine. The abundant immunosuppressive environment promotes both conversion and proliferation of $\mathrm{CD}^{+} \mathrm{CD} 4{ }^{+} \mathrm{CD} 25^{-}$cells into $\mathrm{CD} 4{ }^{+} \mathrm{CD} 25^{+}{ }^{\text {FoxP }} 3^{+}$Tregs population, leading to immune suppression mediated by tumor release of EGFR-containing exosomes (47). Similarly, the increased bioavailability of soluble factors, such as IL-10, TGF- $\beta$, and CCL2, induces both recruitment and accumulation of immunosuppressive cell populations, such as MDSCs, into the TME, resulting in the activation of the signal transducer and transcriptional activator-3 (STAT3) pathway. Furthermore, STAT3 induces the activation of MDSCs, exerting additional immunosuppressive functions, such as inducing impairment of the antigen processing machinery mediated by dendritic cells (DCs), compromising T-cellmediated cytotoxicity, inducing angiogenesis process by VEGF and matrix metalloproteinase (MMP) release, and promoting macrophage phenotype polarization in TAMs. Thus, this TME composition strongly restrains T-cell effector functions through paracrine signals that promote cancer growth (40).

Finally, in addition to the avoiding immune destruction $(56,57)$, a major emerging "hallmark" of immune evasion in TME harboring EGRF mutations is the reprogramming of immunometabolism (58) through CD39/CD73 ectonucleotidases complex that quickly converts adenosine triphosphate (ATP) in adenosine (59), one of most powerful known immunosuppressive metabolite $(18,60)$. Therefore, it is likely that this impairment in the immune system expressed as "uninflamed" TME and characterized by immunological tolerance provokes a weak immunogenicity leading to a poor responsiveness to immunotherapy in patients with EGFR-mutant NSCLC.

\section{IMMUNOMETABOLISM MEDIATED BY CD39/CD73 - ADENOSINE AXIS IN EGFR-MUTANT NSCLC}

The hallmarks of cancer, defined as acquired functional capabilities allowing cancer cells to survive, proliferate, and spread, have been initially identified as modification in cells phenotype enabling replicative immortality, sustained proliferative signaling, evasion from growth suppressors, resistance to cell death, angiogenesis, invasion, and metastasis 
(56). In the last few decades, further hallmarks have been added to this list, including tumor-promoting inflammation, genome instability and mutation, evasion from immune destruction, and reprogramming of energy metabolism (57). During the multi-step tumorigenesis process, the disruption of energy metabolism is proving to be a cardinal feature in lung cancer development and is characterized by the engagement of the aerobic glycolysis, a process where the conversion of glucose into lactate occurs even in presence of sufficient oxygen to support glucose catabolism (Warburg effect) (61).

The TME could alter the immunometabolism and provide immunosuppressive metabolic substrates, thereby modifying the function of immune cells. On the other hands, T-cell subsets need alternative energetic pathways to satisfy their immune response efficiency and to balance the immune system activity. In this context, adenosine signaling involving CD39/CD73 ectonucleotidases expressed on various tumor cells is a critical pathway in TME to evade the immune surveillance and generate an immunosuppressive milieu (18). Specifically, in addition to the pleiotropic effects on immune cells infiltrating tumor, hypoxia directly induces increased adenine nucleotide in TME. Adenosine is an intra- and extra-cellular nucleoside that shows several effects in different tissues depending on its interaction with the following four G-protein-coupled adenosine receptors: A1, A2A, A2B, and A3 (62). In specific, ATP is degraded to adenosine by the ectonucleotidases complexes such as CD39 (ectonucleoside triphosphate diphosphohydrolase-1, E-NTPDase1) and CD73 (ecto-5' -nucleotidase, Ecto5' NTase), which convert ATP to adenosine monophosphate (AMP) and AMP to adenosine and inorganic phosphate, respectively. Adenosine is irreversibly deamidated to inosine by the adenosine deaminase (ADA) enzyme, while extra-cellular adenosine binds to adenosine receptors differently expressed by stromal and immune cells surrounding tumor, thus contributing to immune cell dysfunction (60). Interestingly, A2AR (A2A adenosine receptor) expression has also been found in human lung cancer cells, mostly in adenocarcinoma, exerting a partially direct effect on tumor growth while its antagonism induces tumor growth inhibition through apoptosis activation. Moreover, A2AR is the high-affinity adenosine receptor and appears to have the highest prevalence across all immune cells within both the adaptive and innate systems.

CD73 is endogenously expressed on endothelial cells, epithelial cells, and some immune subsets, and its expression has also been observed in several cancer types, including melanoma, colon, breast, ovarian, and lung cancer (19). Notably, the main drivers of CD73 expression within TME include hypoxia, TGF- $\beta$, type I IFN, IL-1, and prostaglandin (63).

Contrarily, so far little is known about the expression levels of CD39 on intra-tumoral T-cells in NSCLC. Recently, an interesting study reported a consistent co-expression of CD39 and PD-1 receptor on tumor-infiltrating immune cells in NSCLC TME than immune cells from normal lung tissue. CD39 was found also upregulated on several immune cells, including $\mathrm{CD}^{+}$and $\mathrm{CD}^{+}$T-cells, $\mathrm{CD} 16^{+} \mathrm{NK}$ cells, macrophages, and $\mathrm{B}$ cells. Furthermore, $\mathrm{CD} 39^{+} \mathrm{FoxP}^{+}$Tregs were highly enriched in the TME. Therefore, the CD39 upregulation on immune cells in TME suggests that the CD39 pathway may, in addition to $\mathrm{PD}-1$ signaling, represent another relevant mechanism for tumor-induced immunosuppression in NSCLC (64). It is also demonstrated that tumor TGF- $\beta$ induces CD39/CD73 over-expression on MDSCs in NSCLC TME via phosphorylation of $\mathrm{mTOR}$, and subsequently activation of hypoxia-inducible factor-1 (HIF-1) signaling. Thus, TGF$\beta$ stimulating CD39/CD73 expression suppress both T-cells and NKs immunity (65). In addition, the increased expression of CD39 on cytotoxic T-cell induced by MDSCs correlated with poor prognosis in advanced NSCLC patients treated with anti-PD-1 mAbs (66).

Therefore, CD39/CD73 nucleotidases are not only involved in both purine and pyrimidine nucleotide synthesis but represents also a negative modulator of immune signaling through adenosine production in the TME (59). Accordingly, in TME the increased adenosine production mainly through A2AR activation on immune system cells impairs T-cell cytotoxicity, cytokine production, and $\mathrm{T}$ and $\mathrm{NK}$ cell function as well as induces suppression of antigen-presenting cells (APCs) (67). Indeed, in the myeloid compartment, adenosine skews the differentiation of DCs into tolerogenic and immunosuppressive DCs and enhances the immunosuppressive activity of TAM through macrophage M2 polarization. Adenosine release in TME also promotes Tregs and MDSCs proliferation (68) (see Figure 1). Moreover, recent studies have demonstrated that immunosuppressive populations including Tregs and MDSCs enhance their intrinsic suppressive activity also by direct CD73/CD39 upregulating on their cell surface. CD73 expression on FoxP3 ${ }^{+}$Tregs mediates part of their pro-tumorigenic effect converting proinflammatory extracellular ATP into powerful immunosuppressive adenosine (69).

In addition to traditional pathways mediated by CD39/CD73, an alternative enzymatic cascade has been recently reported and includes ectoenzymes such as $\mathrm{CD} 38$ (NAD ${ }^{+}$nucleosidase), CD203 $\alpha$ (ecto-nucleotide pyro-phosphatase phosphodiesterase 1) and $\operatorname{CD73}(70,71)$, thus participating to the composition of the so-called "purinergic milieu" (72). Indeed, in the search of a better understanding of the mechanisms involved in the acquired immunotherapy resistance, it has been reported that the unresponsiveness to PD-1/PD-L1 inhibition in lung murine models is probably mediated by the tumor up-regulation of CD38 enzyme, which is induced by all-trans retinoic acid and IFN- $\beta$ in the TME. Therefore, CD38 represents an alternative ectoenzymatic pathway that limits the cytotoxic activity of $\mathrm{CD} 8^{+}$ T-cells through the activation of adenosine receptor signaling (73), suggesting a potential role of CD38 blockade to overcome immune resistance (74).

Regarding the clinical significance of adenosine signaling, tumor CD73 expression has been widely associated with poor prognosis in several types of cancer including melanoma, colorectal, and triple-negative breast cancers (75). Indeed, to better define the relevance of adenosine signaling in lung cancer, an interesting relationship between immunohistochemistry (IHC) expression of CD73 in tumor tissues and clinical outcome has been found in patients with advanced NSCLC (stage I-III) (76). Moreover, high CD73 expression was an independent factor 
of poor prognosis in terms of OS and recurrence-free survival, thus exhibiting a remarkably worse prognostic meaning (76).

It is also noteworthy the relationship reported by Streicher et al. among the EGFR oncogene activation, the expression of CD73 and the reduced release of IFN- $\gamma$ in NSCLC cell lines compared to wild-type cells. This exploratory analysis was conducted on tumor biopsies of advanced NSCLC patients from a non-randomized phase Ib/II clinical trial (NCT01693562) and from TGCA. According to these evidence, EGFR-mutant adenocarcinomas displayed $>2$-fold increased expression of CD73 compared to wild type, and this mechanism might, at least in part, explain their poor responsiveness to immunotherapy (19). Besides CD73 expression was induced by epidermal growth factor (EGF), and the pharmacological inhibition through EGFRTKi induced its decrease in EGFR-mutated cancer cell lines.

A further study showed for the first time an inverse association between $\mathrm{CD} 73$ expression and activated tumor-infiltrating lymphocytes; in over 1,000 human lung cancer samples (77). It was demonstrated that high levels of CD73 significantly correlated with lower infiltration of activated $\mathrm{CD} 8^{+} \mathrm{T}$-cells compared to those tumor samples with low CD73 expression. Interestingly, CD73 expression was significantly increased in samples with EGFR mutations when compared with wild type tumors (77). To confirm this evidence, it has been recently reported that lower levels of baseline tumor adenosine are associated with a reduced efficacy of anti- PD-1/CTLA-4 mAbs in cohorts of ICI-treated patients (HR $=0.29, P=0.00012)(78)$.

By contrast, Ishii et al. retrospectively showed that high CD73 expression correlated with favorable clinical efficacy of immunotherapy in patients with EGFR-mutated NSCLC who have developed resistance to EGFR-TKIs, although this study had some limitations such as the limited number of patients analyzed (63).

\section{TARGETING IMMUNOSUPPRESSIVE CD73 - ADENOSINE AXIS IN LUNG CANCER: A NEW STRATEGY}

The emerging role of CD39/CD73 - adenosine axis in EGFRmutated NSCLC growth, progression, and ICI resistance has allowed to define a further immune checkpoint as a potential strategy to develop targeted treatments $(79,80)$.

Indeed, regarding the effect of adenosine signaling in the defective regulation of anti-tumor response observed in preclinical tumor models (81-83), different strategies targeting CD73 ectonucleotidase are currently under extensive clinical investigation also in advanced NSCLC (46). Given the broad expression of ectonucleotidases and adenosine receptors in the lung TME, a better understanding of their specific functions will be crucial to implement this new generation of immunological therapeutics (84).

In specific, the adenosine signaling inhibition is based on the use of either small molecule inhibitors or humanized mAbs in order to inhibit adenosine production in the TME or counteract adenosine pathway through targeting the adenosine receptors (85). Small molecules overcame mAbs in terms of both feasibility of administration and bioavailability. By contrast, anti-CD73 mAbs represent a valid alternative for their longer half-life and high specificity. In addition, both direct and indirect effects of mAbs on immune system cells as well as on target cells have been clearly demonstrated $(86,87)$.

Recent studies showed that the single A2AR blockade or the combination with either PD-1/PD-L1 or CTLA-4 mAbs induces T-cell proliferation, enhances the expression of IFN $\gamma$ and granzyme $\mathrm{B}$ by tumor-infiltrating $\mathrm{CD} 8^{+} \mathrm{T}$-cells, thus restraining the tumor growth in preclinical models $(82,83)$. Therefore, the therapeutic effect of A2AR antagonists may be maximized in "inflamed" tumors characterized by infiltrating tumor-reactive $\mathrm{T}$-cells that are otherwise rendered impotent by high adenosine levels in the TME. Interestingly, the A2AR antagonism could prevent negative signaling in T-cells and inhibit angiogenesis process, but also play a direct inhibitory effect on lung cancer cells themselves (88). Anyway, the safety and efficacy of several A2AR inhibitors await evaluation in many ongoing trials also in advanced NSCLC treatment, as summarized in Table 3. Preliminary data demonstrated that A2AR inhibitor (CPI-444), as a single agent and in combination with the anti-PD-L1 mAb (Atezolizumab ${ }^{\circledR}$ ) is well-tolerated and shows anti-tumor activity in refractory renal cancer and NSCLC cohorts (89).

Finally, the current development of anti-CD73-based strategies and relative ongoing clinical trials in monotherapy or combination with immune checkpoint inhibitors as anti-PD(L)1 mAbs or targeted therapies (EGFR-TKIs) in the treatment of advanced NSCLC are summarized in Table 4. The clinical results obtained from these trials might help to clarify the clinical relevance of $\mathrm{CD} 73$ as immune target in the treatment of patients with EGFR-mutated NSCLC.

To date, preliminary results suggest that anti-CD73 mAbs and $\mathrm{PD}-1$ blockade represent a promising approach with an acceptable safety profile in several tumor subtypes such as metastatic melanoma (90), encouraging future therapeutic applications in the clinical practice.

\section{CONCLUSIONS}

Lung tumor remains the most aggressive and life-threatening cancer, although significant therapeutic improvements have been obtained in the last years. To this regard, immunotherapy combinations are promising strategies aimed at restoring the anti-cancer immunity as well as overcoming innate and adaptive resistance to ICIs. In the era of successful immunotherapy, while non-oncogene addicted advanced NSCLC obtains a great survival benefit, the effectiveness of immunotherapy in EGFRmutated NSCLC appears disappointing. Indeed, the anti-PD(L)1 monotherapy has been shown to have minimal activity in EGFR-mutant NSCLC and therefore should only be considered after all other therapies that have been shown to be more effective in this patient population, such as EGFR-TKIs, platinum-doublet chemotherapy, and probably docetaxel plus anti-angiogenic drug (Ramucirumab $\left.{ }^{\circledR}\right)$, have been exhausted.

The identification of additional biomarkers that are predictive of benefit to anti-PD-1 mAbs would be an 
TABLE 3 | Current development status of Adenosine Receptor Antagonists in advanced non-small cell lung cancer.

\begin{tabular}{|c|c|c|c|c|c|c|}
\hline Clinical trial & Drug & Mechanism of action & Pharmaceutical company & $\begin{array}{c}\text { Phase of } \\
\text { development }\end{array}$ & Condition or disease & Drugs combination \\
\hline NCT02503774 & AZD4635 & A2AR antagonist & Medlmmune & $|/| \mid$ & EGFRm NSCLC & $\begin{array}{l}\text { AZD4635; MEDI9447 } \\
\text { (Oleclumab); } \\
\text { Osimertinib }\end{array}$ \\
\hline NCT02403193 & PBF-509 & A2AR antagonist & Palobiofarma SL/Novartis & $|/| \mid$ & $\begin{array}{l}\text { Advanced NSCLC } \\
\text { EGFR L858R/Del19 mutations }\end{array}$ & PBF-509; PDR001 \\
\hline NCT03549000 & NIR178 & A2AR antagonist & Surface oncology, novartis & 1 & $\begin{array}{l}\text { NSCLC; TNBC; PDAC; } \\
\text { Colorectal Cancer MSS; } \\
\text { Ovarian Cancer; } \\
\text { RCC; mCRPC }\end{array}$ & $\begin{array}{l}\text { NIR178; NZV930; } \\
\text { Spartalizumab }\end{array}$ \\
\hline NCT03274479 & PBF-1129 & A2AR antagonist & Palobiofarma SL & 1 & $\begin{array}{l}\text { Locally advanced or } \\
\text { metastatic NSCLC }\end{array}$ & PBF-1129 \\
\hline
\end{tabular}

Source from ClinicalTrials.gov.

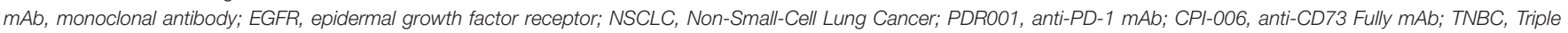

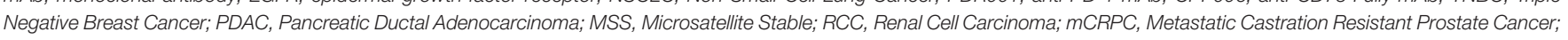
NZV930, anti-CD73 Fully mAb.

TABLE 4 | Current development status of anti-CD73 strategies in advanced non-small cell lung cancer.

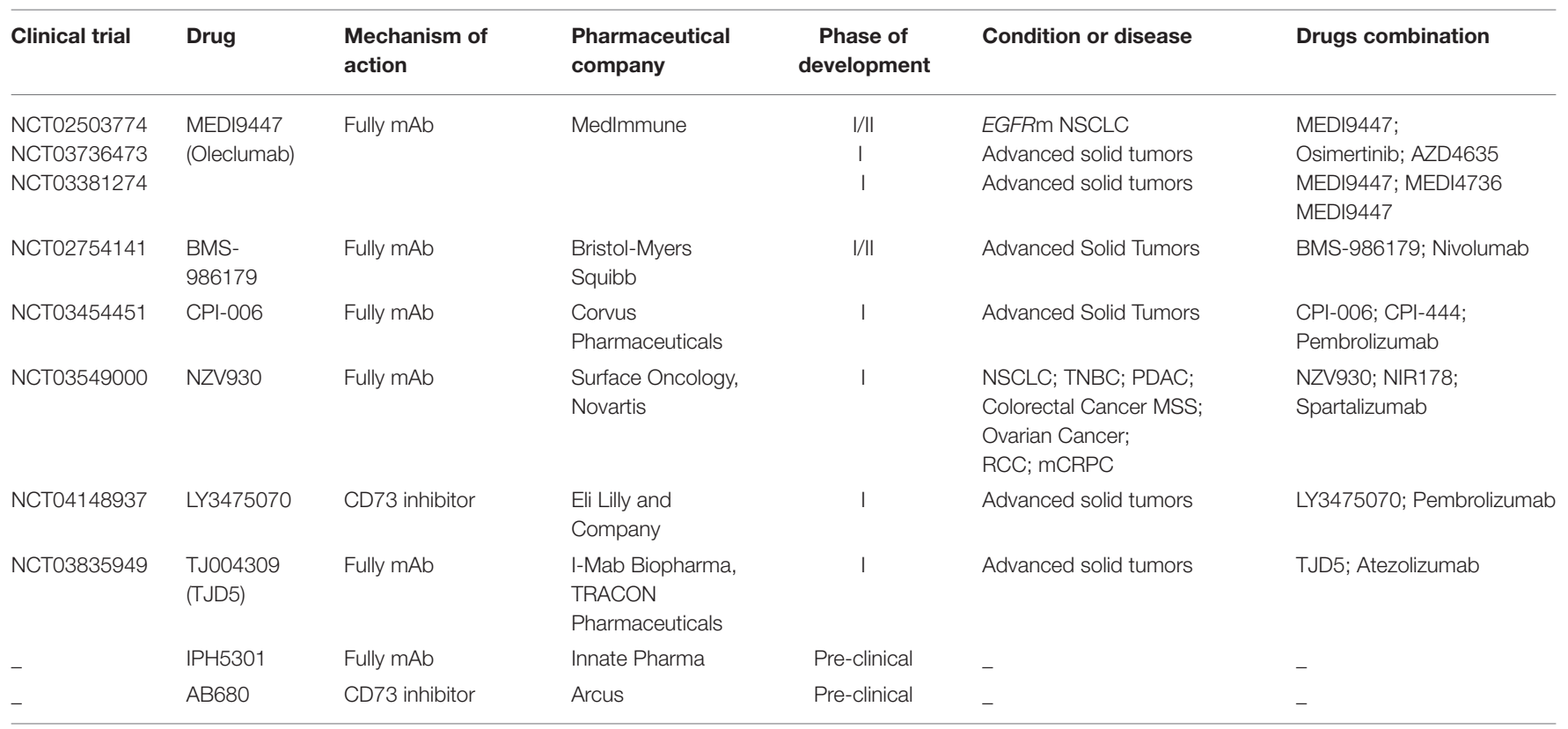

Source from ClinicalTrials.gov.

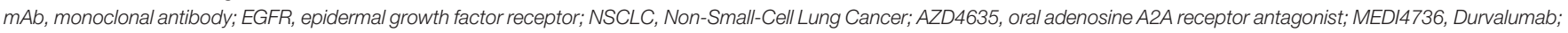


ICRPC, Metastatic Castration Resistant Prostate Cancer; NIR178, oral adenosine A2A receptor antagonist.

important advance in our understanding, especially considering the potential lethal immune-toxicities and high cost of ICIs.

Therefore, a better understanding of biological mechanisms involved in tumor progression and immune evasion as well as of the dynamic hallmarks of EGFR-mutated NSCLC TME is required. The "uninflamed" TME and the low TMB are typical features of EGFR-mutated NSCLC, potentially explaining the impaired response to immunotherapies.

To this regard, an interesting hallmark of NSCLC-harboring oncogenic driver mutations, leading to immune escape, and conferring primary immune resistance, is the reprogramming of energy metabolism mediated by CD39/CD73-adenosine 
signaling. In order to reinforce the critical role of adenosine signaling in tumorigenesis, several trials have demonstrated that CD73 over-expression by cancer cells correlated with poor prognosis in NSCLC patients. Furthermore, in advanced NSCLC recent findings identified a novel relationship between EGFR oncogene activation, over-expression of immunosuppressive molecule such as $\mathrm{CD} 73$ and reduced expression of IFN $\gamma$ signature, and this may explain, at least in part, the limited responsiveness to immunotherapy in EGFR-mutated NSCLC. Despite these preliminary evidence, the exact relevance of CD73adenosine signaling in both EGFR-mutated tumor cells and infiltrating immune cells to the efficacy of immune checkpoint inhibitors remains unclear. Anyway, in the preclinical studies the pharmacological antagonism of CD39/CD73-adenosine signaling potentiated anti-tumor responses in preclinical models that otherwise failed to respond to anti-PD-1/PD-L1 inhibition.

\section{REFERENCES}

1. Siegel RL, Miller KD, Jemal A. Cancer statistics, 2018. CA Cancer J Clin. (2018) 68:7-30. doi: 10.3322/caac.21442

2. Jemal A, Bray F, Center MM, Ferlay J, Ward E, Forman D. Global cancer statistics. Cancer J Clin. (2011) 61:69-90. doi: 10.3322/caac.20107

3. Ferlay, J, Soerjomataram I, Dikshit R, Eser S, Mathers C, Rebelo $\mathrm{M}$, et al. Cancer incidence and mortality worldwide: sources, methods and major patterns in GLOBOCAN 2012. Int $J$ Cancer. (2015) 1:E359-86. doi: 10.1002/ijc. 29210

4. Dong J, Lin B, Zhou Q, Huang D. Advances in targeted therapy and immunotherapy for non-small cell lung cancer based on accurate molecular typing. Front Pharmacol. (2019) 10:230. doi: 10.3389/fphar.2019.00230

5. Herbst, RS, Morgensztern D, Boshoff C. The biology and management of nonsmall cell lung cancer. Nature. (2018) 553:446-454. doi: 10.1038/nature25183

6. Takeda M, Nakagawa K. First- and second-generation EGFR-TKIs are all replaced to osimertinib in chemo-naïve EGFR mutation-positive non-small cell lung cancer? Int J Mol Sci. (2019) 20:146. doi: 10.3390/ijms20010146

7. De Marinis F, Barberis M, Barbieri V, Marchianò A, Gasperini S, Migliorino $\mathrm{MR}$, et al. Diagnosis and first-line treatment of non-small cell lung cancer in the era of novel immunotherapy: recommendations for clinical practice. Exp Rev Respirat Med. (2019) 13:217-28. doi: 10.1080/17476348.2019.1569517

8. Planchard D, Popat S, Kerr K, Novello S, Smit EF, Faivre-Finn C, et al. Metastatic non-small cell lung cancer. ESMO Ann Oncol. (2019) 29(Suppl. 4):iv192-237. doi: 10.1093/annonc/mdy275

9. Ramalingam SS, Vansteenkiste J, Planchard D, Cho BC, Gray JE, Ohe $\mathrm{Y}$, et al. Overall survival with osimertinib in untreated, EGFR-mutated advanced NSCLC. N Engl J Med. (2020) 382:41-50. doi: 10.1056/NEJMoa19 13662

10. Gridelli C, Casaluce F. Immunotherapies in the management of epidermal growth factor receptor mutated non-small cell lung cancer: a role will be found? Transl Lung Cancer Res. (2018) 7(Suppl. 4):S370-2. doi: 10.21037/tlcr.2018.09.18

11. Ruiz-Patino A, Arrieta O, Cardona AF, Martin C, Raez LE, Zatarain-Barron $\mathrm{ZL}$, et al. Immunotherapy at any line of treatment improves survival in patients with advanced metastatic non-small cell lung cancer (NSCLC) compared with chemotherapy (Quijote-CLICaP). Thorac Cancer. (2020) 11:353-61. doi: 10.1111/1759-7714.13272

12. Lisberg A, Cummings A, Goldman JW, Bornazyan K, Reese N, Wang T, et al. A phase II study of pembrolizumab in EGFR-mutant, PD-L1+, tyrosine kinase inhibitor naïve patients with advanced NSCLC. J Thorac Oncol. (2018) 13:1138-45. doi: 10.1016/j.jtho.2018.03.035

13. Hastings K, Yu HA, Wei W, Sanchez-Vega F, DeVeaux M, Choi J, et al. EGFR mutation subtypes and response to immune checkpoint blockade
These data agree with observations that blockade of other immune checkpoint inhibitors over-expressed in the TME, such as CTLA-4, TIM-3, TIGIT can enhancing IFN $\gamma$ production by $\mathrm{CD}^{+}$TILs following anti-PD-1 inhibition.

In conclusion, adenosinergic signaling is emerging as a powerful immune-metabolic checkpoint in advanced NSCLC. Hence, further pre-clinical data and clinical trials aiming at translating adenosine signaling inhibition strategies in EGFR-mutated NSCLC are needed to target the dysregulated immunometabolism in the TME, in order to overcome primary resistance to immunotherapy.

\section{AUTHOR CONTRIBUTIONS}

All authors listed have made a substantial, direct and intellectual contribution to the work, and approved it for publication. treatment in non-small-cell lung cancer. Ann Oncol. (2019) 30:131120. doi: 10.1093/annonc/mdz141

14. Jia Y, Li X, Jiang $\mathrm{T}$, Zhao $\mathrm{C}$, Zhang L, Liu $\mathrm{X}$, et al. EGFR-targeted therapy alters the tumor microenvironment in EGFR-driven lung tumors: implications for combination therapies. Int J Cancer. (2019) 145:143244. doi: 10.1002/ijc.32191

15. Yang JC, Shepherd FA, Kim DW, Lee GW, Lee JS, Chang GC, et al. Osimertinib plus durvalumab versus osimertinib monotherapy in EGFR T790M-positive NSCLC following previous EGFR-TKI therapy: CAURAL brief report. J Thorac Oncol. (2019) 14:933-9. doi: 10.1016/j.jtho.2019.02.001

16. Yang JC, Gadgeel SM, Sequist LV, Wu CL, Papadimitrakopoulou VA, Su WC, et al. Pembrolizumab in combination with Erlotinib or Gefitinib as first-line therapy for advanced NSCLC with sensitizing EGFR mutation. J Thorac Oncol. (2019) 14:553-9. doi: 10.1016/j.jtho.2018.11.028

17. Dong ZY, Zhang JT, Liu SY, Su J, Zhang C, Xie Z, et al. EGFR mutation correlates with uninflamed phenotype and weak immunogenicity, causing impaired response to PD-1 blockade in non-small cell lung cancer. Oncoimmunology. (2017) 6:e1356145. doi: 10.1080/2162402X.2017.1356145

18. Passarelli A, Tucci M, Mannavola F, Felici C, Silvestris F. The metabolic milieu in melanoma: role of immune suppression by CD73/adenosine. Tumour Biol. (2019) 42:1010428319837138. doi: 10.1177/1010428319837138

19. Streicher K, Higgs BW, Wu S, Coffman K, Damera G, Durham N, et al. Increased CD73 and reduced IFNG signature expression in relation to response rates to anti-PD-1(L1) therapies in EGFR-mutant NSCLC. J Clin Oncol. (2017) 35:abstr 11505. doi: 10.1200/JCO.2017.35.15_suppl.11505

20. Martinez P, Peters S, Stammers T, Soria JC. Immunotherapy for the first-line treatment of patients with metastatic non-small cell lung cancer. Clin Cancer Res. (2019) 25:2691-8. doi: 10.1158/1078-0432.CCR-18-3904

21. Postow MA, Sidlow R, Hellmann MD. Immune-related adverse events associated with immune checkpoint blockade. N Engl J Med. (2018) 378:15868. doi: 10.1056/NEJMra1703481

22. Stucci S, Palmirotta R, Passarelli A, Silvestris E, Argentiero A, Lanotte L, et al. Immune- related adverse events during anticancer immunotherapy: pathogenesis and management. Oncol Lett. (2017) 14:5671-80. doi: 10.3892/ol.2017.6919

23. Tucci M, Passarelli A, Todisco A, Mannavola F, Stucci LS, D'Oronzo $\mathrm{S}$, et al. The mechanisms of acute interstitial nephritis in the era of immune checkpoint inhibitors in melanoma. Ther Adv Med Oncol. (2019) 11:1758835919875549. doi: 10.1177/1758835919875549

24. Passarelli A, Mannavola F, Stucci LS, Tucci M, Silvestris F. Immune system and melanoma biology: a balance between immuno- surveillance and immune escape. Oncotarget. (2017) 8:106132-42. doi: 10.18632/oncotarget.22190

25. Tucci M, Passarelli A, Mannavola F, Felici C, Stucci SL, Cives M, et al. Immune system evasion as hallmark of melanoma progression: the role of dendritic cells. Front Oncol. (2019) 9:1148. doi: 10.3389/fonc.2019.01148 
26. Pardoll DM. The blockade of immune checkpoints in cancer immunotherapy. Nat Rev Cancer. (2012) 12:252-64. doi: 10.1038/nrc3239

27. Reck M, Rodriguez-Abreu D, Robinson AG, Hui R, Csoszi T, Fulop A, et al. Pembrolizumab versus chemotherapy for PD-L1-positive non-small-cell lung cancer. N Engl J Med. (2016) 375:1823-33. doi: 10.1056/NEJMoa1606774

28. Gandhi L, Rodriguez-Abreu D, Gadgeel S, Esteban E, Felip E, De Angelis F, et al. Pembrolizumab plus chemotherapy in metastatic non-small-cell lung cancer. N Engl J Med. (2018) 378:2078-92. doi: 10.1056/NEJMoa1801005

29. Socinsky MA, Jotte RM, Cappuzzo F, Orlandi F, Stroyakovskiy MD, Nogami $\mathrm{N}$, et al. Atezolizumab for first-line treatment of metastatic nonsquamous NSCLC. N Engl J Med. (2018) 378:2288-301. doi: 10.1056/NEJMoa1716948

30. Herbst, RS, Baas P, Kim DW, Felip E, Pérez-Gracia JL, Han $\mathrm{J}-\mathrm{Y}$, et al. Pembrolizumab versus docetaxel for previously treated, PD-L1-positive, advanced non-small-cell lung cancer (KEYNOTE-010): a randomised controlled trial. Lancet. (2016) 387:1540-50. doi: 10.1016/S0140-6736(15)01281-7

31. Brahmer J, Reckamp KL, Baas P, Crinò L, Eberhart WE, Poddubskaya E, et al. Nivolumab versus docetaxel in advanced squamous-cell non-small-cell lung cancer. N Engl J Med. (2015) 373:1-13. doi: 10.1056/NEJMoa1504627

32. Rittmeyer A, Barlesi F, Waterkamp D, Park K, Ciardiello F, von Pawel J, et al. Atezolizumab versus docetaxel in patients with previously treated non-small-cell lung cancer (OAK): a phase 3, open-label, multicentre randomised controlled trial. Lancet. (2017) 389:255-65. doi: 10.1016/S0140-6736(16)32517-X

33. Borghaei H, Paz-Ares L, Horn L, Spigel DR, Steins M, Ready NE, et al. Nivolumab versus docetaxel in advanced nonsquamous non-smallcell lung cancer. N Engl J Med. (2015) 373:162739. doi: 10.1056/NEJMoal 507643

34. Berghoff AS, Bellosillo B, Caux C, de Langen A, Mazieres J, Normanno $\mathrm{N}$, et al. Immune checkpoint inhibitor treatment in patients with oncogene-addicted non-small cell lung cancer (NSCLC): summary of a multidisciplinary round-table discussion. ESMO Open. (2019) 4:e000498. doi: 10.1136/esmoopen-2019-000498

35. Lee CK, Man J, Lord S, Links M, Gebski V, Mok T, et al. Checkpoint inhibitors in metastatic EGFR-mutated non-small cell lung cancer - a meta-analysis. $J$ Thoracic Oncol. (2016) 12:403-7. doi: 10.1016/j.jtho.2016.10.007

36. Lee CK, Man J, Lords S, Cooper W, Links M, Gebski V, et al. Clinical and molecular characteristics associated with survival among patients treated with checkpoint inhibitors for advanced non-small cell lung carcinoma. a systematic review and meta-analysis. JAMA Oncol. (2018) 4:2106. doi: 10.1001/jamaoncol.2017.4427

37. Lisberg A, Garon EB. Lack of clearly defined role for anti-programmed death-(ligand) 1 therapy in epidermal growth factor receptor mutated non-small cell lung cancer. Transl Lung Cancer Res. (2019) 8:1957. doi: $10.21037 /$ tlcr.2019.02.01

38. Masuda K, Horinouchi H, Tanaka M, Higashiyama R, Shinno Y, Sato J, et al. Efficacy of immune-checkpoint inhibitors and EGFR-TKIs in NSCLC patients with high PD-L1 expression. J Thoracic Oncol. (2019) 14:S2923 doi: $10.1016 /$ j.jtho.2019.08.588

39. Akbay EA, Koyama S, Carretero J, Altabef A, Tchaicha JH, Christensen $\mathrm{CL}$, et al. Activation of the PD-1 pathway contributes to immune escape in EGFR-driven lung tumors. Cancer Discov. (2013) 3:135563. doi: 10.1158/1535-7163.TARG-13-B290

40. Lin A, Wei T, Meng H, Luo P, Zhang J. Role of the dynamic tumor microenvironment in controversies regarding immune checkpoint inhibitors for the treatment of non-small cell lung cancer (NSCLC) with EGFR mutations. Mol Cancer. (2019) 18:139. doi: 10.1186/s12943-019-1062-7

41. Reck M, Shankar G, Lee A, Coleman S, McCleland M, Papadimitrakopoulou VA, et al. Atezolizumab in combination with bevacizumab, paclitaxel and carboplatin for the first-line treatment of patients with metastatic non- squamous non-small cell lung cancer, including patients with EGFR mutations. Expert Rev Respir Med. (2019) 14:125-6. doi: 10.1080/17476348.2020.1701439

42. Im JS, Herrmann AC, Bernatchez C, Haymaker C, Molldrem JJ, Hong WK, et al. Immune-modulation by epidermal growth factor receptor inhibitors: implication on anti-tumor immunity in lung cancer. PLOS ONE. (2016) 11:e0160004. doi: 10.1371/journal.pone.0160004
43. Ribas A, Lawrence D, Atkinson V, Agarwal S, Miller WH, Carlino MS, et al. Combined BRAF and MEK inhibition with PD-1 blockade immunotherapy in BRAF-mutant melanoma. Nat Med. (2019) 25:936940. doi: 10.1038/s41591-019-0476-5

44. Oxnard GR, Yang JC, Yu H, Kim SW, Saka H, Horn L, et al. TATTON: a multiarm, phase Ib trial of osimertinib combined with selumetinib, savolitinib, or durvalumab in EGFR-mutant lung cancer. Ann Oncol. (2020) 31:50716. doi: 10.1016/j.annonc.2020.01.013

45. Gibbons DL, Chow LQ, Kim DW, Kim TY, Song X, Jiang R, et al. Efficacy, safety and tolerability of MEDI4736 (durvalumab [D]), a human IgG1 antiprogrammed cell death-ligand-1 (PD- L1) antibody, combined with gefitinib (G): a phase I expansion in TKI-naive patients (pts) with EGFR mutant NSCLC. J Thorac Oncol. (2016) 11:S79. doi: 10.1016/S1556-0864(16)30171-X

46. Santaniello A, Napolitano F, Servetto A, De Placido P, Silvestris N, Bianco C, et al. Tumour microenvironment and immune evasion in EGFR addicted NSCLC: hurdles and possibilities. Cancers. (2019) 11:E1419. doi: 10.3390/cancers11101419

47. Huang SH, Li Y, Zhang J, Rong J, Ye S. Epidermal growth factor receptorcontaining exosomes induce tumor-specific regulatory T cells. Cancer Investig. (2013) 31:330-5. doi: 10.3109/07357907.2013.789905

48. Mannavola F, Tucci M, Felici C, Passarelli A, D’Oronzo S, Silvestris F. Tumor-derived exosomes promote the in vitro osteotropism of melanoma cells by activating the SDF1/CXCR4/CXCR7 axis. J Transl Med. (2019) 17:230. doi: 10.1186/s12967-019-1982-4

49. Teng MW, Ngiow SF, Ribas A, Smyth MJ. Classifying cancers based on T-cell infiltration and PD-L1. Cancer Res. (2015) 75:2139-45. doi: 10.1158/0008-5472.CAN-15-0255

50. Toki MI, Mani N, Smithy JW, Liu Y, Altan M, Wasserman B, et al. Immune marker profiling and programmed death ligand 1 expression across NSCLC mutations. J Thorac Oncol. (2018) 13:1884-96. doi: 10.1016/j.jtho.2018.09.012

51. Pollack BP, Sapkota B, Cartee TV. Epidermal growth factor receptor inhibition augments the expression of MHC class I and II genes. Clin Cancer Res. (2011) 17:4400-13. doi: 10.1158/1078-0432.CCR-10-3283

52. Takada K, Toyokawa G, Tagawa T, Kohashi K, Shimokawa M, Akamine $\mathrm{T}$, et al. PD-L1 expression according to the EGFR status in primary lung adenocarcinoma. Lung Cancer. (2018) 116:1-6. doi: 10.1016/j.lungcan.2017.12.003

53. Rizvi H, Sanchez-Vega F, La K, Chatila W, Jonsson P, Halpenny D, et al. Molecular determinants of response to anti-programmed cell death (PD)-1 and anti-programmed death-ligand 1 (PD-L1) blockade in patients with non-small-cell lung cancer profiled with targeted next-generation sequencing. J Clin Oncol. (2018) 36:633-41. doi: 10.1200/JCO.2017.7 5.3384

54. Update on the Phase III NEPTUNE trial of Imfinzi Plus Tremelimumab in Stage IV Non-Small Cell Lung Cancer [press release]. AstraZeneca. (2019). Available online at: https://bit.ly/2Zdd5Zu (accessed August 21, 2019).

55. Spigel DR, Schrock AB, Fabrizio D, Frampton GM, Sun J, He J, et al. Total mutation burden (TMB) in lung cancer (LC) and relationship with response to PD-1/PD-L1 targeted therapies. J Clin Oncol. (2016) 34(Suppl. 15):9017. doi: 10.1200/JCO.2016.34.15_suppl.9017

56. Hanahan D, Weinberg RA. The hallmarks of cancer. Cell. (2000) 100:5770. doi: 10.1016/S0092-8674(00)81683-9

57. Hanahan D, Weinberg RA. Hallmarks of cancer: the next generation. Cell. (2011) 144:646-74. doi: 10.1016/j.cell.2011.02.013

58. Chang C-H, Pearce EL. Emerging concepts of T cell metabolism as a target of immunotherapy. Nat Immunol. (2016) 17:364-8. doi: 10.1038/ni.3415

59. Allard B, Longhi MS, Robson SC, Stagg J. The ectonucleotidases CD39 and CD73: novel checkpoint inhibitor targets. Immunol Rev. (2017) 276:12144. doi: 10.1111/imr.12528

60. Stagg J, Smyth MJ. Extracellular adenosine tripho- sphate and adenosine in cancer. Oncogene. (2010) 29:5346-58. doi: 10.1038/onc. 2010.292

61. Singer K, Cheng WC, Kreutz M, Ho PC, Siska PJ. Immunometabolism in cancer at a glance. Dis Model Mech. (2018) 11:dmm034272. doi: 10.1242/dmm.034272

62. Fredholm BB, IJzerman AP, Jacobson KA, Linden J, Muller CE. International union of basic and clinical pharmacology. LXXXI. nomenclature and 
classification of adenosine receptors - an update. Pharmacol Rev. (2011) 63:1-34. doi: 10.1124/pr.110.003285

63. Ishii $H$, Azuma $K$, Kawahara A, Kinoshita $T$, Matsuo N, Naito $\mathrm{Y}$, et al. Predictive value of CD73 expression for the efficacy of immune checkpoint inhibitors in NSCLC. Thoracic Cancer. (2020) 11:950-5. doi: 10.1111/1759-7714.13346

64. Tøndell A, Wahl Freim SG, Sponaas A-M, Sorhaug S, Borset M, Haug M. Ectonucleotidase CD39 and checkpoint signalling receptor programmed death 1 are highly elevated in intratumoral immune cells in non-small-cell lung cancer. Transl Oncol. (2020) 13:17-24. doi: 10.1016/j.tranon.2019.09.003

65. Li J, Wang L, Chen X, Li L, Yu Li Y, Ping Y, et al. CD39/CD73 upregulation on myeloid-derived suppressor cells via TGF- $\beta$-mTOR-HIF-1 signaling in patients with non-small cell lung cancer. Oncoimmunology. (2017) 6:e1320011. doi: 10.1080/2162402X.2017.1320011

66. Koh J, Lee KY, Kim B, Kim MS, Cho HJ, Sun J-M, et al. Abstract A138: CD39 increase on cytotoxic T-cell induced by myeloid-derived suppressor cell correlated with poor prognosis in patients with non-small cell lung cancer. Cancer Immunol Res. (2019) 7(Suppl. 2):A138. doi: 10.1158/2326-6074.CRICIMTEATIAACR18-A138

67. Ohta A. A metabolic immune checkpoint: adenosine in tumor microenvironment. Front Immunol. (2016) 7:109. doi: 10.3389/fimmu.2016.00109

68. Antonioli L, Colucci R, La Motta C, Tuccori M, Awwad O, Da Settimo F, et al. Adenosine deaminase in the modulation of immune system and its potential as a novel target for treatment of inflammatory disorders. Curr Drug Targets. (2012) 13:842-62 doi: 10.2174/138945012800564095

69. Stagg J, Divisekera U, Duret H, Sparwasser T, Teng MWL, Darcy PK, et al. CD73-deficient mice have increased antitumor immunity and are resistant to experimental metastasis. Cancer Res. (2011) 71:2892900. doi: 10.1158/0008-5472.CAN-10-4246

70. Horenstein AL, Chillemi A, Zaccarello G, Bruzzone S, Quarona V, Zito A, et al. A CD38/CD203a/CD73 ectoenzymatic pathway independent of CD39 drives a novel adenosinergic loop in human T lymphocytes. Oncoimmunology. (2013) 2:e26246. doi: 10.4161/onci.26246

71. Horenstein AL, Chillemi A, Zini R, Quarona V, Bianchi N, Manfredini R, et al. Cytokine-induced killer cells express CD39, CD38, CD203a, CD73 ectoenzymes and P1 adenosinergic receptors. Front Pharmacol. (2018) 9:196. doi: 10.3389/fphar.2018.00196

72. Wo YJ, Gan ASP, Lim X, Tay ISY, Lim S, Lim JCT et al. The roles of CD38 and CD157 in the solid tumor microenvironment and cancer immunotherapy. Cells. (2020) 9:26. doi: 10.3390/cells9010026

73. Chen L, Diao L, Yang Y, Yi X, Rodriguez BL, Li Y, et al. CD38mediated immuno- suppression as a mechanism of tumor cell escape from PD-1/PD-L1 blockade. Cancer Discov. (2018) 8:1156-175. doi: 10.1158/2159-8290.CD-17-1033

74. Mittal D, Vijayan D, Smyth MJ. Overcoming acquired PD1/PD-L1 resistance with CD38 blockade. Cancer Discov. (2018) 8:1066-8. doi: 10.1158/2159-8290.CD-18-0798

75. Monteiro I, Vigano S, Faouzi M, Treilleux I, Michielin O, Ménétrier-Caux C, et al. CD73 expression and clinical significance in human metastatic melanoma. Oncotarget. (2018) 9:26659-69. doi: 10.18632/oncotarget.25426

76. Inoue $\mathrm{Y}$, Yoshimura $\mathrm{K}$, Kurabe $\mathrm{N}$, Kahyo $\mathrm{T}$, Kawase $\mathrm{A}$, Tanahashi $\mathrm{M}$, et al. Prognostic impact of $\mathrm{CD} 73$ and $\mathrm{A} 2 \mathrm{~A}$ adenosine receptor expression in non-small-cell lung cancer. Oncotarget. (2017) 8:873851. doi: 10.18632/oncotarget.14434

77. Lee Chun P, Kyunghoon R, Won BK, Cho A, Song A, Anker $\mathrm{JF}$, et al. Immunologic and clinical implications of CD73 expression in non-small cell lung cancer (NSCLC). J Clin Oncol. (2018) 36(Suppl. 15):12050. doi: 10.1200/JCO.2018.36.15_suppl. 12050
78. Sidders B, Zhang P, Goodwin K, O’Connor G, Russel DL, Borodovsky A, et al. Adenosine signaling is prognostic for cancer outcome and has predictive utility for immunotherapeutic response. Clin Cancer Res. (2020) 26:217687. doi: 10.1158/1078-0432.CCR-19-2183

79. Prado-Garcia H, Sanchez-Garcia FJ. Editorial: immunometabolism in tumor microenvironment. Front Immunol. (2017) 8:374. doi: 10.3389/fimmu.2017.00374

80. Boison D, Yegutkin GG. Adenosine metabolism: emerging concepts for cancer therapy. Cancer Cell. (2019) 36:582-96. doi: 10.1016/j.ccell.2019.10.007

81. Willingham SB, Ho PY, Hotson A, Hill C, Piccione EC, Hsieh J, et al. A2AR antagonism with CPI-444 induces antitumor responses and augments efficacy to anti-PD-(L)1 and anti-CTLA-4 in preclinical models. Cancer Immunol Res. (2018) 6:1136-49. doi: 10.1158/2326-6066.CIR-18-0056

82. Beavis PA, Milenkovski N, Henderson MA, John LB, Allard B, Loi S, et al. Adenosine receptor 2A blockade increases the efficacy of anti-PD-1 through enhanced antitumor t-cell responses. Cancer Immunol Res. (2015) 3:50617. doi: 10.1158/2326-6066.CIR-14-0211

83. Allard B, Pommey S, Smyth MJ, Stagg J. Targeting CD73 enhances the antitumor activity of anti-PD-1 and anti-CTLA-4 mAbs. Clin Cancer Res. (2013) 19:5626-35. doi: 10.1158/1078-0432.CCR-13-0545

84. Stagg J, Divisekera U, McLaughlin N, Sharkey J, Pommey S, Denoyer D, et al. Anti-CD73 antibody therapy inhibits breast tumor growth and metastasis. Proc Natl Acad Sci USA. (2010) 107:1547-52. doi: 10.1073/pnas.0908801107

85. Allard D, Chrobak P, Allard B, Messaoudi N, Stagg J. Targeting the CD73-adenosine axis in immuno-oncology. Immunol Lett. (2019) 205:319. doi: 10.1016/j.imlet.2018.05.001

86. Perrot I, Michaud HA, Giraudon-Paoli M, Augier S, Docquier A, Gros L, et al. Blocking antibodies targeting the CD39/CD73 immunosuppressive pathway unleash immune responses in combination cancer therapies. Cell Rep. (2019) 27:2411-25. doi: 10.1016/j.celrep.2019.04.091

87. Hay CM, Sult E, Huang Q, Mulgrew K, Fuhrmann SR, McGlinchey KA, et al. Targeting CD73 in the tumor microenvironment with MEDI9447. Oncoimmunology. (2016) 5:e1208875. doi: 10.1080/2162402X.2016.1208875

88. Mediavilla-Varela M, Luddy K, Noyes D, Khalil FK, Neuger AM, Soliman $\mathrm{H}$, et al. Antagonism of adenosine A2A receptor expressed by lung adenocarcinoma tumor cells and cancer associated fibroblasts inhibits their growth. Cancer Biol Ther. (2013) 14:9:860-8. doi: 10.4161/cbt.25643

89. Fong L, Forde PM, Powderly JD, Goldman JW, Nemunaitis JJ, Luke JJ, et al. Safety and clinical activity of adenosine A2a receptor (A2aR) antagonist, CPI-444, in anti PD1/PDL1 treatment-refractory renal cell (RCC) and nonsmall cell lung cancer (NSCLC) patients. J Clin Oncol. (2017) 35:(Suppl. 15):3004. doi: 10.1200/JCO.2017.35.15_suppl.3004

90. Lannone R, Miele L, Maiolino P, Pinto A, Morello S. Adenosine limits the therapeutic effectiveness of anti-CTLA4 mAb in a mouse melanoma model. Am J Cancer Res. (2014) 4:172-81.

Conflict of Interest: CG received honoraria as speaker bureau and advisory board member from Astra Zeneca, BMS, MSD, and Roche.

The remaining authors declare that the research was conducted in the absence of any commercial or financial relationships that could be construed as a potential conflict of interest.

Copyright (c) 2020 Passarelli, Aieta, Sgambato and Gridelli. This is an open-access article distributed under the terms of the Creative Commons Attribution License (CC $B Y)$. The use, distribution or reproduction in other forums is permitted, provided the original author(s) and the copyright owner(s) are credited and that the original publication in this journal is cited, in accordance with accepted academic practice. No use, distribution or reproduction is permitted which does not comply with these terms. 